\title{
Article \\ Clinical, Genetic and Functional Characterization of a Novel AVPR2 Missense Mutation in a Woman with X-Linked Recessive Nephrogenic Diabetes Insipidus
}

\author{
Senthil Selvaraj ${ }^{1}$, Dírcea Rodrigues ${ }^{2}$, Navaneethakrishnan Krishnamoorthy ${ }^{1} \mathbb{D}$, Khalid A. Fakhro ${ }^{1,3,4} \mathbb{D}$, \\ Luís R. Saraiva $1,4,5, *$ (D) and Manuel C. Lemos ${ }^{6, *(\mathbb{D})}$ \\ 1 Department of Genetics, Sidra Medicine, Doha P.O. Box 26999, Qatar; sselvaraj@sidra.org (S.S.); \\ nkrishnamoorthy2@sidra.org (N.K.); kfakhro@sidra.org (K.A.F.) \\ 2 Serviço de Endocrinologia, Diabetes e Metabolismo, Centro Hospitalar Universitário de Coimbra, \\ 3000-075 Coimbra, Portugal; dircearodrigues@chuc.min-saude.pt \\ 3 Department of Genetic Medicine, Weill Cornell Medical College, Doha P.O. Box 24144, Qatar \\ 4 College of Health and Life Sciences, Hamad Bin Khalifa University, Doha P.O. Box 24144, Qatar \\ 5 Monell Chemical Senses Center, 3500 Market Street, Philadelphia, PA 19104, USA \\ 6 CICS-UBI, Health Sciences Research Centre, University of Beira Interior, 6200-506 Covilhã, Portugal \\ * Correspondence: saraivalmr@gmail.com (L.R.S.); mclemos@fcsaude.ubi.pt (M.C.L.)
}

check for

updates

Citation: Selvaraj, S.; Rodrigues, D.; Krishnamoorthy, N.; Fakhro, K.A.; Saraiva, L.R.; Lemos, M.C. Clinical, Genetic and Functional Characterization of a Novel AVPR2 Missense Mutation in a Woman with X-Linked Recessive Nephrogenic Diabetes Insipidus. J. Pers. Med. 2022, 12, 118. https://doi.org/10.3390/ jpm12010118

Academic Editor: Giovanna Floridia

Received: 8 December 2021

Accepted: 5 January 2022

Published: 17 January 2022

Publisher's Note: MDPI stays neutral with regard to jurisdictional claims in published maps and institutional affiliations.

Copyright: (C) 2022 by the authors. Licensee MDPI, Basel, Switzerland. This article is an open access article distributed under the terms and conditions of the Creative Commons Attribution (CC BY) license (https:// creativecommons.org/licenses/by/ $4.0 /)$.

\begin{abstract}
Nephrogenic diabetes insipidus (NDI) is a rare disorder characterized by renal unresponsiveness to the hormone vasopressin, leading to excretion of large volumes of diluted urine. Mutations in the arginine vasopressin receptor-2 (AVPR2) gene cause congenital NDI and have an X-linked recessive inheritance. The disorder affects almost exclusively male family members, but female carriers occasionally present partial phenotypes due to skewed inactivation of the X-chromosome. Here, we report a rare case of a woman affected with X-linked recessive NDI, presenting an average urinary output of $12 \mathrm{~L} /$ day. Clinical and biochemical studies showed incomplete responses to water deprivation and vasopressin stimulation tests. Genetic analyses revealed a novel heterozygous missense mutation (c.493G > C, p.Ala165Pro) in the AVPR2 gene. Using a combination of in-silico protein modeling with human cellular models and molecular phenotyping, we provide functional evidence for phenotypic effects. The mutation destabilizes the helical structure of the AVPR2 transmembrane domains and disrupts its plasma membrane localization and downstream intracellular signaling pathways upon activation with its agonist vasopressin. These defects lead to deficient aquaporin 2 (AQP2) membrane translocation, explaining the inability to concentrate urine in this patient.
\end{abstract}

Keywords: vasopressin; AVPR2; nephrogenic diabetes insipidus; genetics; case report

\section{Introduction}

Water homeostasis in the body is maintained by balancing thirst-stimulated water intake and renal water excretion. Urine volume and concentration is regulated by the action of vasopressin (or antidiuretic hormone), which is secreted into the bloodstream by the posterior lobe of the pituitary gland in response to extracellular fluid hypertonicity [1] Deficiencies in vasopressin secretion or renal unresponsiveness to vasopressin lead to central diabetes insipidus or nephrogenic diabetes insipidus (NDI), respectively [2]. Both diseases are characterized by excessive urine production (polyuria) and water intake (polydipsia). An inappropriately dilute urine with a high serum osmolality, assessed through a water-deprivation test, establishes the diagnosis of diabetes insipidus [2]. NDI can be further distinguished from central diabetes insipidus by the failure to normalize urine osmolality upon stimulation with the vasopressin synthetic analogue, desmopressin (1-deamino-8-D-arginine vasopressin, dDAVP) [3].

Congenital NDI is a rare disorder that is usually inherited as an X-linked recessive trait due to mutations in the arginine vasopressin receptor 2 (AVPR2) gene [4-6] or, less 
frequently, as an autosomal recessive or dominant trait due to mutations in the aquaporin-2 $(A Q P 2)$ gene [7]. These genetic forms of NDI are usually present since birth and can result in life-threatening dehydration and neurologic impairment if water intake is inadequate [8,9]. The AVPR2 gene is located on the $\mathrm{X}$-chromosome (region $\mathrm{Xq} 28$ ), consists of three coding exons and encodes a 371-amino acid G protein-coupled receptor (GPCR) that contains seven transmembrane domains $[10,11]$. AVPR2 is expressed at the basolateral membrane of the principal cells of the collecting duct in the kidneys, where water reabsorption takes place. The binding of vasopressin to AVPR2 in the kidney activates adenylyl cyclase, increasing intracellular cyclic adenosine monophosphate (cAMP), which in turn activates cAMPdependent protein kinase. This activation leads to the phosphorylation and trafficking of the AQP2 channel, followed by insertion of AQP2 along the apical cell membrane of the collecting duct, thereby allowing water to enter the cell and reducing renal excretion of water [7].

To date, at least 280 different loss-of-function mutations in AVPR2 have been reported in patients with NDI (Human Gene Mutation Database, www.hgmd.org, accessed on 5 January 2022). In vitro functional studies have shown that mutant receptors are either improperly transcribed, retained in the endoplasmic reticulum, or reach the cell surface, but are unable to properly bind vasopressin or to trigger an intracellular cAMP signal [12]. As the mode of inheritance is X-linked recessive, affected individuals are mainly males who are hemizygous for the mutations. While heterozygous females are usually unaffected, some females may exhibit variable degrees of polyuria and polydipsia due to skewed inactivation of the X-chromosome [13].

This study aimed to investigate the genetic defect and its functional consequences in a woman affected with NDI. Using targeted sequencing analysis, we identified a novel heterozygous missense mutation in $A V P R 2$, which causes a substitution of the alanine (Ala) to proline (Pro) at residue position 165 (Ala165Pro). We then tested the impact of this mutation on the protein structure using an in-silico approach and assessed its functional consequences by using in vitro human cell models.

\section{Material and Methods}

\subsection{Clinical Studies}

A 26-year-old Portuguese woman presented a history of polyuria and polydipsia. She reported that her symptoms had been present since infancy. Despite significant polyuria (approximately $12 \mathrm{~L} /$ day), she had adapted to her long-standing condition and never sought medical treatment. Her past medical history had no other relevant occurrences. Family history included four affected males (father, great-uncle, uncle and cousin) (Figure 1A), who declined investigation. Physical examination was unremarkable. We performed a water-deprivation and dDAVP stimulation test for the differential diagnosis of diabetes insipidus [2,3]. Starting in the morning, all fluid intake was stopped and body weight, urine output and plasma and urine osmolarity were measured hourly until the patient lost $5 \%$ of her body weight. At this time, fluid intake was resumed to prevent further dehydration and a $2 \mu \mathrm{g}$ intramuscular injection of dDAVP was administered, followed by hourly measurements of urine osmolarity. The cardiovascular and coagulation responses to dDAVP were determined on a different day [14]. An intravenous line with a three-way stopcock was inserted in one arm while the patient was supine and baseline blood samples collected at -30 and $0 \mathrm{~min}$. The patient then received an infusion of dDAVP $(0.3 \mu \mathrm{g} / \mathrm{kg}$, in $100 \mathrm{~mL}$ saline, over $20 \mathrm{~min}$ ) and additional blood samples collected at 30, 60, 90 and $120 \mathrm{~min}$. Blood pressure and heart rate were recorded every $10 \mathrm{~min}$ from -30 to $0 \mathrm{~min}$, every $5 \mathrm{~min}$ from 0 to $30 \mathrm{~min}$ and every $10 \mathrm{~min}$ from 30 to $120 \mathrm{~min}$. Blood samples were centrifuged immediately after collection and the plasma separated for measurements of factor VIIIc, von Willebrand factor and renin activity [14]. 
A Pedigree of the NDI patient

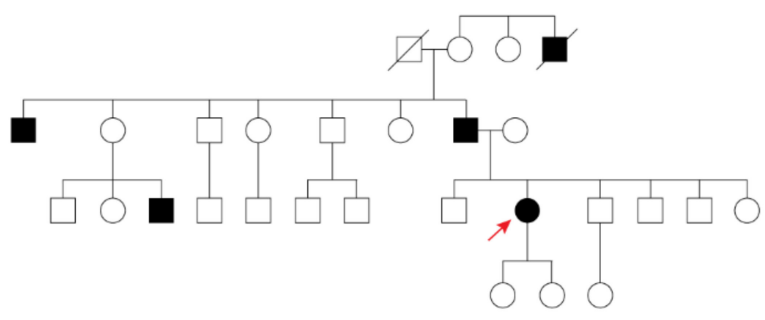

B Urine output and body weight

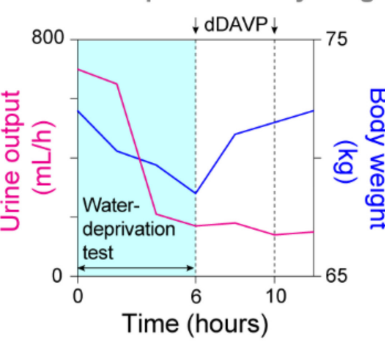

C Serum and urine osmolality

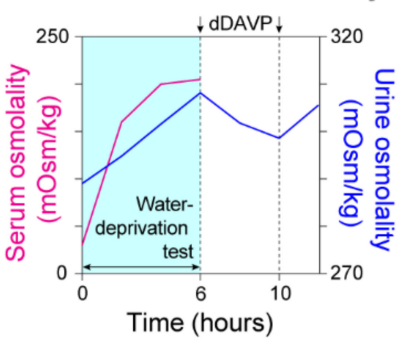

Hemodynamic and coagulation responses

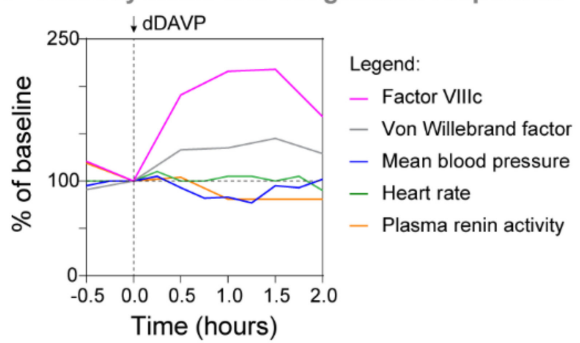

E Response to treatment

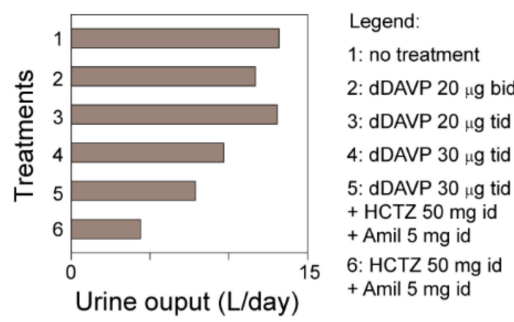

F AVPR2 genomic structure and the PCR amplified fragments

H Restriction enzyme analysis of DNA

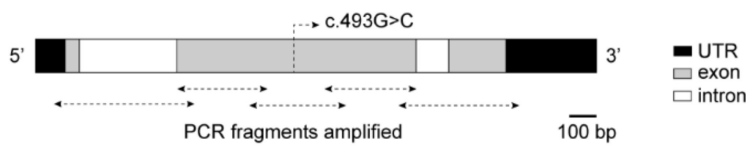

G Identification of the Ala165Pro mutation in AVPR2
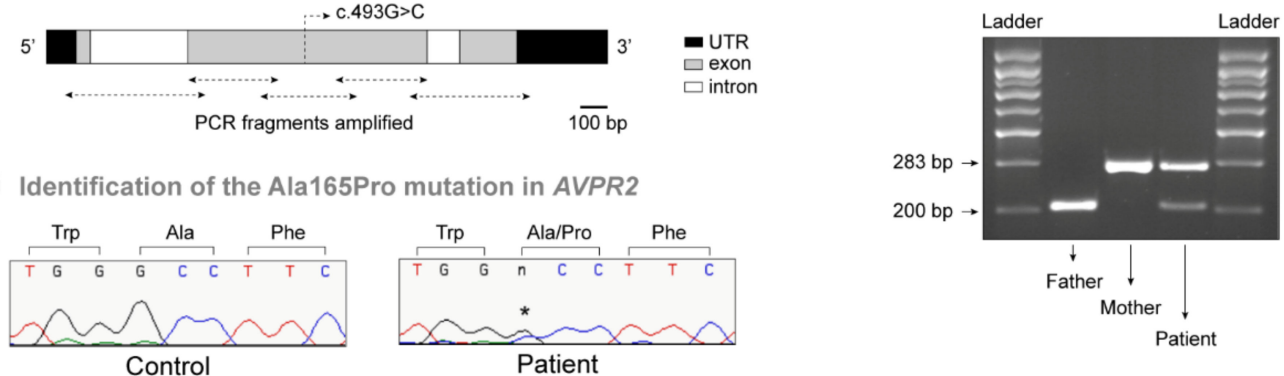

Figure 1. Biochemical and genetic studies of the patient with nephrogenic diabetes insipidus (NDI) (A) Pedigree of the patient with NDI. The inheritance follows a typical X-linked recessive pattern, apart from the reported female patient (red arrow). Individuals are represented as males (squares), females (circles), affected (filled symbol), unaffected (open symbol) and deceased (oblique line through symbol). (B,C) Water-deprivation and dDAVP stimulation tests. The water-deprivation test was continued until the patient lost $5 \%$ of her body weight, which occurred after $6 \mathrm{~h} \mathrm{(B).} \mathrm{At} \mathrm{the}$ end of the test, serum osmolality had increased from 276 to $311 \mathrm{mOsm} / \mathrm{Kg}$ (normal 275-295) and the urine osmolality had increased from 95 to only $191 \mathrm{mOsm} / \mathrm{Kg}$ (normal 300-900) (C). Arrows indicate the administration of dDAVP $(2 \mu \mathrm{g}$ intramuscular injection), which did not increase urine osmolality, thereby supporting the diagnosis of NDI. (D) Extra-renal responses to dDAVP. Arrow indicates the administration of $\operatorname{dDAVP}(0.3 \mu \mathrm{g} / \mathrm{kg}$, intravenous infusion over $20 \mathrm{~min})$. Mean arterial blood pressure decreased 18\% (normal $>10 \%$ ), heart rate increased 10\% (normal $>20 \%$ ), plasma renin activity increased $4 \%$ (normal $>65 \%$ ), factor VIIIc increased $2.2 \times($ normal $>3 \times$ ), von Willebrand factor increased $1.5 \times$ (normal $>2 \times)$. (E) Response to treatment. Treatment with increasing doses of dDAVP did not significantly improve the polyuria. Treatment with hydrochlorothiazide (HCTZ) $50 \mathrm{mg}$ bid, amiloride chloridrate (Amil) $5 \mathrm{mg}$ bid and a low-sodium diet, resulted in a reduction of diuresis to approximately $5 \mathrm{~L} /$ day. (F) Genomic structure (Xq28) showing the exons (grey boxes), introns (white boxes), $3^{\prime}$ - and $5^{\prime}$ - untranslated regions (UTR) (black boxes) of the AVPR gene. The locations of the PCR amplified fragments and the identified mutation are shown. (G) Partial DNA sequencing of exon 2 of the AVPR2 gene in the patient and a control. We identified a novel heterozygous missense mutation (c.493G > C, p.Ala165Pro; noted by an asterisk) in the patient. (H) Agarose gel electrophoresis of MwoI-digested PCR fragments. The mutation creates a restriction site for this enzyme, resulting in fragments of 200 base pairs (bp) and $83 \mathrm{bp}$ (not shown), whereas the normal allele results in fragments of $283 \mathrm{bp}$. The patient is heterozygous for the mutation, the father is hemizygous and the mother is homozygous for the normal allele. We used a $100 \mathrm{bp}$ ladder as a DNA size marker. 


\subsection{Genetic Studies}

The genetic studies were approved by the Institutional Research Ethics Committee of the Faculty of Health Sciences at the University of Beira Interior, Portugal (study reference number CE-FCS-2013-017) and written informed consent obtained from the patient. Genomic DNA was extracted from peripheral blood leukocytes of the patient and used with polymerase chain reaction (PCR) primers to amplify the coding regions of the AVPR2 gene (primer sequences and PCR conditions are available upon request). We performed bidirectional sequencing of the PCR products using an automated capillary DNA sequencer (GenomeLab TM GeXP, Genetic Analysis System, Beckman Coulter, Fullerton, CA, USA) and the identified genetic variant was validated by restriction enzyme analysis with MwoI. The parents of the patient accepted genetic testing and were screened for the presence of the variant by restriction enzyme analysis. To determine if the identified genetic variant was present in the general population [15], we searched the Genome Aggregation Database (gnomAD) [16] and the 1000 Genomes Project. Bioinformatic programs (PolyPhen-2, SIFT, PROVEAN and MutationTaster) [17] were used to predict the consequences of the variant on protein function. The variant was analyzed by VarSome [18] and classified according to American College of Medical Genetics and Genomics (ACMG) criteria [19]. We based the nomenclature of the variant on the AVPR2 cDNA reference sequence (GenBank accession number NM_000054.6), whereby nucleotide c.1 was the A of the ATG-translation initiation codon.

\subsection{Molecular Modeling of AVPR2}

As the human AVPR2 lacks a 3D structure from experimental studies (such as X-ray crystallography or nuclear magnetic resonance), we performed the molecular modeling of its protein 3D structure to assess the impact of the Ala165Pro substitution. Previously reported modeling of AVPR2 was based on the amino acid replacement method with another computer model of AVPR1 [20,21]. After the release of this model, the X-ray structures and structure-function studies of multiple other related $\mathrm{G}$ protein-coupled receptors (PDB ID: 5WQC, 6TQ4, 6TP3, 6U1N, 6TP6, 6TPK, 6TPJ, 6V9S) were released [22-27]. We chose the X-ray structure of the oxytocin receptor (PDB ID: 6TPK) as the template for the AVPR2 model building due to its sequence and structural conservation (44\% identity, 58\% similarity), similar fold, domain distribution and shared key binding sites.

We used the template specification method to model the human AVPR2 in Swissmodel [28]. The quality of the model was estimated using the recently developed distance constraints method [29]. Structural analyses, secondary structural observations, binding regions and GPCR-family such as conserved residues were mapped using PyMOL (The PyMOL Molecular Graphics System, Schrödinger LLC, New York, NY, USA) as previously described [30]. The structure of the Ala165Pro-AVPR2 mutant was predicted using the structure generated with the wild-type AVPR2 sequence as a template and local network analysis of the intra-molecular interactions in the wild-type and Ala165Pro substitution was carried out as previously described [31].

\subsection{Cell Culture and Transfection}

HEK293 cells (ATCC) were grown in Gibco Dulbecco's Modified Eagle Medium (DMEM, Thermo Fischer Scientific, Waltham, MA, USA) containing 10\% fetal bovine serum (FBS, Thermo Fischer Scientific, Waltham, MA, USA), $100 \mathrm{U} / \mathrm{mL}$ penicillin and $100 \mu \mathrm{g} / \mathrm{mL}$ streptomycin at $37^{\circ} \mathrm{C}$ in a humidified atmosphere with $5 \% \mathrm{CO}$. Plasmids pcDNA 3.1 (+) WT-AVPR2-GFP, A165P-AVPR2-GFP, WT-AVPR2-HA, A165P-AVPR2-HA and AQP2 were purchased from Genscript Inc (Piscataway, NJ, USA). For the transient transfection, we used Lipofectamine 3000 (Thermo Fischer Scientific, Waltham, MA, USA) according to the manufacturer's instructions. At $36 \mathrm{~h}$ after transfection, the cells were used for the downstream analysis. 


\section{5. cAMP and PKA Activity Assays}

The level of cAMP in AVPR2 transfected HEK293 cells was measured using a cAMP direct immunoassay kit as described by the manufacturer's instructions (Abcam, Cambridge, UK). Briefly, the day before transfection, HEK293 cells were plated in a 6-well plate at around $70-80 \%$ confluency. Cells were transfected with WT-AVPR2 or A165P-AVPR2 at the concentration of $2.5 \mu \mathrm{g}$ per well using Lipofectamine 3000. At $36 \mathrm{~h}$ after transfection, cells were stimulated with or without AVPR2 agonist dDAVP. Cells were washed with PBS, lysed using $0.1 \mathrm{M} \mathrm{HCl}$ and the cell lysates were centrifuged at $14,000 \times g$ for $10 \mathrm{~min}$. The supernatant was used to measure the cAMP levels and the ELISA plate was read at OD $540 \mathrm{~nm}$.

We measured the cellular activity of PKA using a non-radioactive PKA kinase assay kit according to the manufacturer's instructions (Abcam, Cambridge, UK). PKA measurement in cells was based on a solid phase enzyme-linked immuno-absorbent assay (ELISA) that utilizes a specific synthetic peptide as a substrate for PKA and a polyclonal antibody that recognizes the phosphorylated form of the substrate. HEK293 cells were transfected with WT-AVPR2 or A165P-AVPR2 in a 6-well plate as described above. Cells were lysed with lysis buffer and the activity of PKA was measured at $450 \mathrm{~nm}$.

\subsection{Western Blotting and Cell Surface Biotinylation Assay}

We used the Mem-PER Plus Membrane Protein Extraction Kit (Thermo Fischer Scientific, Waltham, MA, USA) to isolate the crude membrane from cells expressing WT and A165P-AVPR2. Briefly, cells were treated with mild detergent to solubilize the cytosolic protein. The cytosolic fraction was isolated by centrifugation and the remaining cell pellet was treated with a second detergent to solubilize the membrane protein and centrifuged to collect the membrane fraction. Cell surface biotinylation was performed using the Pierce Cell Surface Biotinylation and Isolation Kit (Thermo Fischer Scientific, Waltham, MA, USA) according to the manufacturer's instruction. Briefly, the surface proteins were labeled by incubating the cells with EZ-Link Sulfo-NHS-SS-Biotin at $4{ }^{\circ} \mathrm{C}$. The cells were lysed and the labeled protein with debris removed by centrifugation $\left(12,000 \times g, 15 \mathrm{~min}, 4^{\circ} \mathrm{C}\right)$. Supernatant fractions $(500 \mu \mathrm{L})$ were incubated with protein-A Sepharose, preincubated with rabbit polyclonal anti-HA antibody and incubated overnight at $4{ }^{\circ} \mathrm{C}$. Beads were washed twice with $250 \mu \mathrm{L}$ of lysis buffer. The crude membrane and cell surface biotinylated samples were resolved in 4-12\% Nupage gel and transferred into a Polyvinylidene Fluoride (PVDF) membrane. The PVDF membrane was blocked with non-fat milk buffer and incubated overnight with respective primary antibody at $4{ }^{\circ} \mathrm{C}$, followed by HRP-conjugated secondary antibody incubation. The resolved proteins were visualized using Chemdoc (Bio-Rad Laboratories, Hercules, CA, USA) and bands were quantified by densitometric analysis using Image (NIH, Bethesda, MD, USA), as previously described [32].

\subsection{Immunofluorescence}

Immunofluorescence was used to visualize the cell surface expression of GFP-tagged AVPR2. HEK293 cells were transfected either with GFP tagged- WT-AVPR2 or A165P-AVPR2. Cell surface receptor staining was performed on live cells by incubation with a rabbit polyclonal anti-GFP (Thermo Fischer Scientific, Waltham, MA, USA) primary antibody diluted in DMEM with $1 \%$ BSA) for $1 \mathrm{~h}$ at $4{ }^{\circ} \mathrm{C}$. Cells were then washed with ice-cold phosphate-buffered saline (PBS) and fixed with 3\% PFA for $15 \mathrm{~min}$ at room temperature followed by incubation with a Alexa Fluor 546-conjugated (Thermo Fischer Scientific, Waltham, MA, USA) secondary antibody.

\subsection{Statistical Analysis}

Data analysis was performed using GraphPad Prism 8.0.0 software (GraphPad, San Diego, CA, USA). Statistical comparisons were made using unpaired $t$-tests with Welch's correction (two-tail) or one-way ANOVAs (Tukey multiple comparisons corrections). Ex- 
perimental values are displayed as mean \pm SEM. Differences in the mean values were considered to be significant at $p<0.05$.

\section{Results}

\subsection{Blood and Urine Biochemical Tests}

To confirm the diagnosis of NDI, we performed a water-deprivation and dDAVP stimulation test (see Methods). The basal urine output of the patient was $12.3 \mathrm{~L} /$ day, with specific gravity 1.003 (normal 1.010-1.030) and osmolality $98 \mathrm{mOsm} / \mathrm{kg}$ (normal range 300-900). Basal serum chemistry results, including electrolytes, were normal. The water-deprivation test was stopped after $6 \mathrm{~h}$ when the patient lost $5 \%$ of her body weight (Figure 1B,C). At this time, the serum osmolality had increased from 276 to $311 \mathrm{mOsm} / \mathrm{Kg}$ (normal range: $275-295 \mathrm{mOsm} / \mathrm{Kg}$ ) and the urine osmolality had increased from 95 to only $191 \mathrm{mOsm} / \mathrm{Kg}$ (normal range: 300-900 mOsm/Kg) (Figure 1C). To test the response to vasopressin, we administered a $2 \mu \mathrm{g}$ intramuscular injection of dDAVP at hour 6 and repeated $4 \mathrm{~h}$ later. No increase in urine osmolality was observed (Figure 1C), consistent with renal unresponsiveness to vasopressin. We also determined the cardiovascular and coagulation responses to an intravenous infusion of dDAVP (see Methods) [2,3]. Mean arterial blood pressure decreased 18\% (normal $>10 \%$ ), heart rate increased $10 \%$ (normal $>20 \%$ ), plasma renin activity increased $4 \%$ (normal $>65 \%$ ), factor VIIIc increased $2.2 \times($ normal $>3 \times)$, von Willebrand factor increased $1.5 \times$ (normal $>2 \times$ ), thus, showing only partial responses to dDAVP (Figure 1D). To confirm the resistance to vasopressin, the patient was treated with increasing doses of dDAVP, which did not significantly improve the polyuria (Figure 1E). Finally, the patient was put on treatment with hydrochlorothiazide $50 \mathrm{mg}$ bid, amiloride chloridrate $5 \mathrm{mg}$ bid and low-sodium diet, resulting in a reduction of diuresis to approximately $5 \mathrm{~L} /$ day (Figure 1E).

\subsection{Identification of a Novel Missense Mutation in AVPR2}

Next, we performed DNA amplification and sequencing of the AVPR2 gene in the patient (Figure 1F). We identified a heterozygous missense variant in exon 2, which predicted the substitution of an Alanine by a Proline at amino acid position 165 of the protein (chrX:153,171,453 G > C; NM_000054.6: c.493G > C; p.Ala165Pro) (Figure 2B). Restriction enzyme analysis with MwoI confirmed the presence of the variant in the patient and in her father and its absence in her mother (Figure 1G). The variant was absent in both the gnomAD and the 1000 Genomes Project population databases and was predicted to be damaging by multiple in silico prediction tools (PolyPhen-2, SIFT, PROVEAN and MutationTaster). The variant fulfilled the ACMG criteria for "Likely Pathogenic" (PM1, PM2, PP2, PP3).

Taken together, these results confirm the diagnosis of $X$-linked recessive NDI in this female patient and identified a novel AVPR2 missense mutation (Ala165Pro) involved in this disease.

\subsection{The Missense Ala165Pro Variant Disrupts AVPR2 Function}

Using molecular modeling tools (see Methods), we first assembled the model structure of the 'wild-type' human AVPR2 (Ala165), which includes the seven transmembrane domains/helices (H1-7) connected with three intracellular (I1-3) and extracellular (E1-3) loops, typical of GPCRs (Figure 2A). The Ala165 residue sits in the middle of helix 4, surrounded by the vasopressin binding pocket (Figure 2B), which includes a key GPCR signature residue (Trp164) [22,33]. Helix 4 also contains other residues (Lys100, Ala110, Met120, Leu175, Arg202, Phe307) suggested to play a role in shaping the vasopressin binding pocket and its affinity to its ligand [20] (Figure 2C). 
A Wild-type AVPR2 3D model

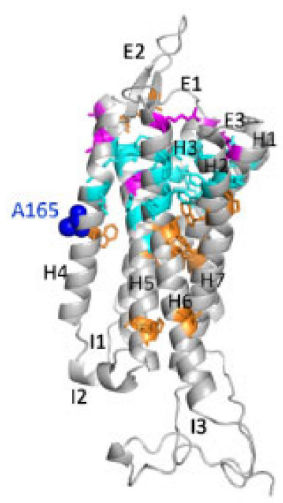

D Mutant AVPR2 3D model

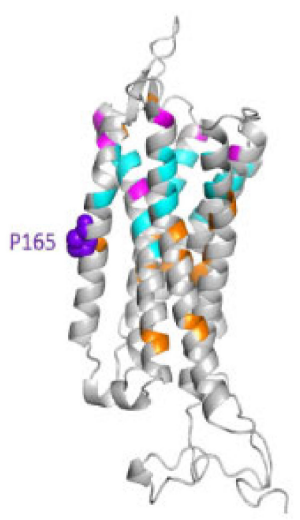

\section{B AVP binding pocket of AVPR2}

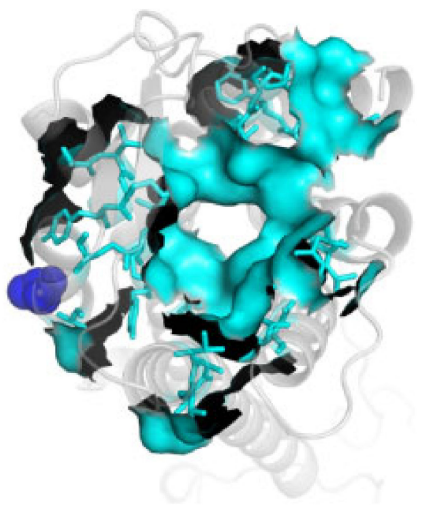

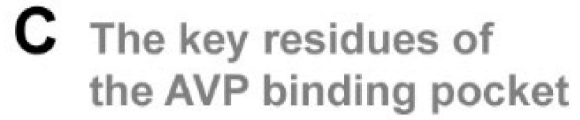

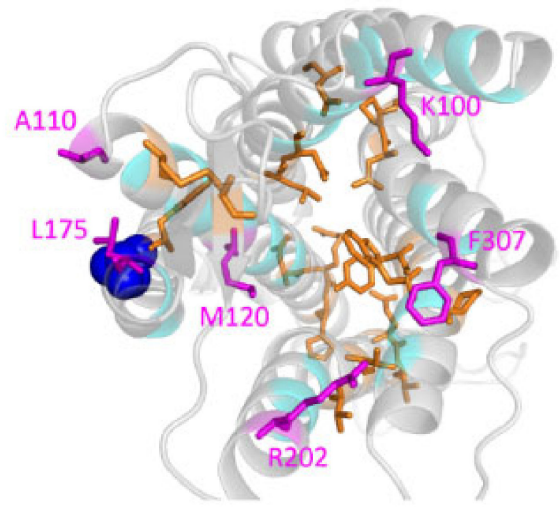

\section{E Molecular interaction networks in the wild-type (WT) and the mutant (Ala165Pro) AVPR2}
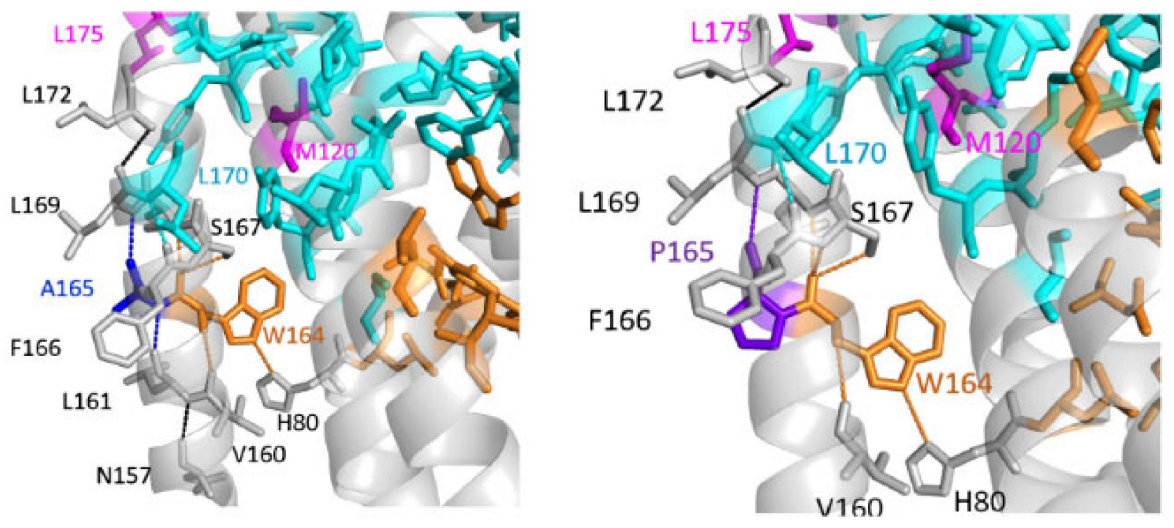

Figure 2. Model structure with key regions of human AVPR2 and Ala165Pro-AVPR2. (A) Lateral view of the 3D protein model of the wild-type human AVPR2, including seven transmembrane domains/helices (H1-7) connected with three intracellular (I1-3) and extracellular (E1-3) loops. Dark blue sphere: Alanine residue at position 165 (A165). (B) Surface mapping of the vasopressin (AVP) ligand-binding pocket in AVPR2. (C) Key residues in the AVP binding region and conserved residues of rhodopsin-like GPCR family. (D) Lateral view of the 3D protein model of the mutant Ala165ProAVPR2. (E) Molecular interaction networks around residue 165 in the WT-AVPR (left panel) and the mutant Ala165Pro-AVPR2 (right panel). Dark blue sphere: Alanine (A) residue at position 165 (A165); Purple blue sphere: Proline (P) residue at position 165 (P165); Cyan sticks and surface: AVP binding pocket; Orange sticks: conserved residues; Magenta sticks: -binding residues; Grey sticks: other residues in the network; Black dash: hydrogen bonds. Residues are indicated by their position number, preceded by the corresponding amino-acid letter: A, Alanine; F, Phenylalanine; H, Histidine; L, Leucine; M, Methionine; N, Asparagine; P, Proline; S, Serine; V, Valine; W, Tryptophan.

Next, we generated the model structure of the 'mutant' human AVPR2 (Figure 2D). The mutant Ala165Pro substitution is in the middle of a block of ten hydrophobic residues (Val Leu Val Ala Trp Ala Phe Ser Leu Leu Leu) that provide stability to helix 4 and results in side-chain chemical structure from hydrophobic (Ala) to an uncharged polar ring (Pro) which can introduce a break in the hydrophobicity. To test this assumption, we performed an analysis of the local network of intra-molecular interactions connected with the residue at position 165 . We identified a hydrogen bond 
between Ala165 and Leu161 in the wild-type AVPR2 (Figure 2E, left panel), which does not exist between Pro165 and Leu161 in the mutant AVPR2 (Figure 2F, right panel). The lack of this interaction disconnects the network of 165-161-157 in helix 4, potentially destabilizing the helical structure. Indeed, Ala to Pro mutations in transmembrane helices were previously shown to break and/or distort the helical structure [34,35]. Moreover, another AVPR2 missense substitution in position 165, Ala165Asp, has previously been reported to interfere with proper receptor trafficking [36].

Together, these results reveal that the Ala165Pro missense mutation disrupts the intramolecular interactions and destabilizes the helical structure, which possibly affects AVPR2 trafficking, stability and function.

To further investigate the functional effects of the Ala165Pro substitution on AVPR2, we performed a series of in vitro cellular assays. In these assays, we generated HA-tagged and GFP-tagged versions of AVPR2 containing the wild-type (WT-AVPR2) and the missense mutation (Ala165Pro-AVPR2) identified in the patient and expressed them in HEK293 cells. We found no significant differences between the total protein levels of the wild-type and mutant AVPR2 (Figure 3A). In contrast, the amount of AVPR2 protein present in the crude plasma membrane extracts was significantly reduced in the mutant compared to the wild-type (Figure 3B). Consistent with these results, cell surface biotinylation experiments revealed that the cell surface expression of AVPR2 was also significantly impaired in the mutant compared to the wild-type (Figure 3C). Immunofluorescence experiments provided the third line of evidence for reduced membrane levels of the mutant AVPR2 compared to the wild-type (Figure 3D).

Taken together, these data show that the Ala165Pro mutation does not compromise overall receptor abundance but reduces the plasma membrane levels of AVPR2.

\subsection{Biological Impact of the Mutant AVPR2}

Vasopressin activates the basolateral membrane of AVPR2 in renal collecting duct principal cells, leading to the activation of the Gas-cAMP-PKA signaling cascade and the subsequent phosphorylation and membrane translocation of AQP2 water channels to initiate water reabsorption [37]. To understand whether the Ala165Pro substitution in AVPR2 affect its function, we first evaluated its ability to generate cAMP and PKA kinase activation in response to dDAVP compared to the wild-type. Upon stimulation with dDAVP, we observed significant increases in the levels of cAMP and PKA kinase activation in cells transfected with WT-AVPR2 and Ala165Pro-AVPR2, but these increases were significantly higher in the wild-type compared to the mutant (Figure 4A,B).

Stimulation of AVPR2 by dDAVP activates the PKA kinase by phosphorylating it at Thr197 and inhibiting the phosphorylation of p38-MAPK [37]. Our experiments further revealed that dDAVP stimulation increased PKA kinase phosphorylation in cells transfected with WT-AVPR2 and Ala165Pro-AVPR2, but these increases were only significant in the wild-type (Figure 4C). In contrast, we observed increased p38-MAPK phosphorylation in cells transfected with the mutant Ala165Pro-AVPR2 compared to the WT-AVPR2.

Next, to study the impact of A165P mutant on AQP2 membrane translocation, we performed cell surface biotinylation experiments after co-transfection with AQP2 and the wild-type or mutant versions of AVPR2. These experiments revealed that upon stimulation with dDAVP, the plasma membrane translocation of AQP2 was significantly increased in WT-AVPR2 but not in the mutant Ala165Pro-AVPR2 (Figure 4E).

Taken together, these data show that the Ala165Pro mutation causes a decrease in cAMP levels and PKA kinase activation in response to dDAVP and subsequently impairs the translocation of AQP2 to the plasma membrane. 
A Whole cell lysate

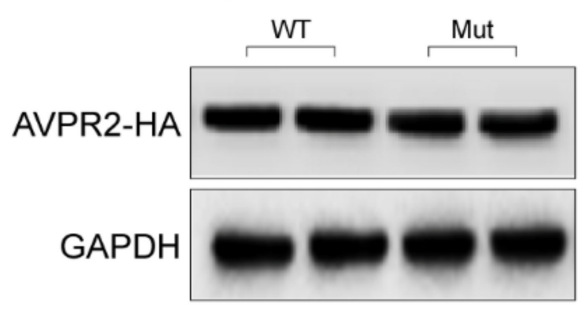

B Crude plasma membrane extracts

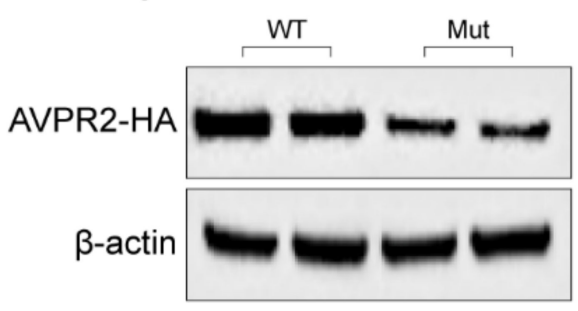

C Cell surface biotinylation

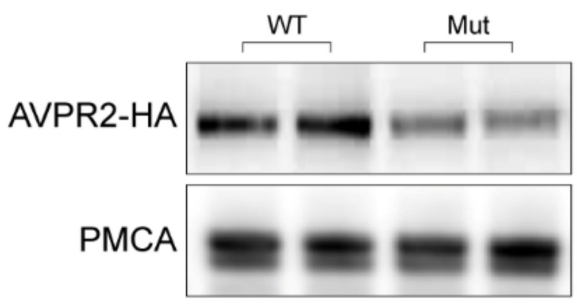

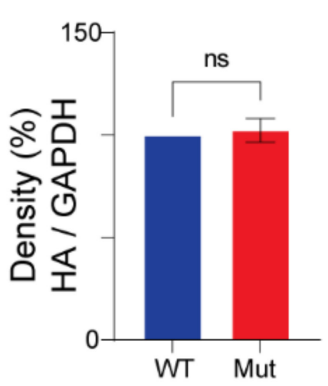
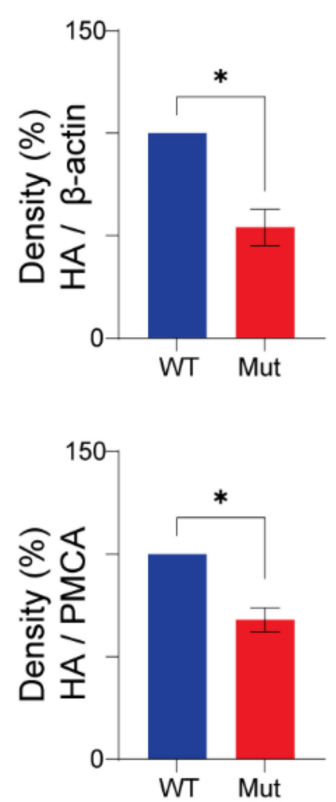

D

Immunofluorescence of AVPR2-GFP
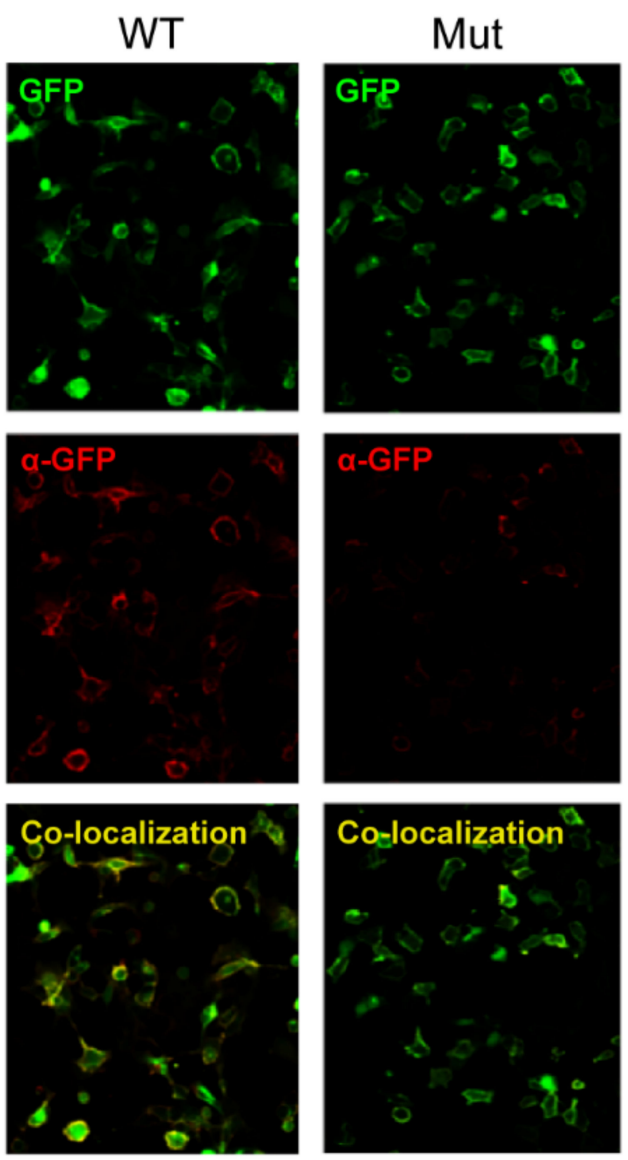

Figure 3. A165P mutant impairs the cell surface localization of AVPR2. (A) HEK293 cells were transfected with HA-tagged WT-AVPR2 (WT) or Ala165Pro-AVPR2 (Mut). At $36 \mathrm{~h}$ after transfection, cells were harvested, lysed and proteins were analyzed by immunoblotting (left panel). Glyceraldehyde 3-phosphate dehydrogenase (GAPDH) was used as a loading control. Semiquantitative analysis of the effects of the Ala165Pro mutation on the total levels of AVPR2 protein was performed by densitometry (right panel). (B) Crude plasma membrane extracts were prepared from cells transfected with HA-tagged WT or Mut AVPR2 and proteins were analyzed by immunoblotting (left panel). $\beta$-actin was used as a loading control. A semiquantitative analysis of the effects of Ala165Pro mutation on the plasma membrane levels of AVPR2 protein was performed by densitometry (right panel). (C) In parallel, to analyze the cell surface expression of AVPR2, HA-tagged WT or Mut AVPR2 expressing cells were biotinylated using Sulfo-NHS-SS-Biotin and then precipitated with streptavidin-Sepharose beads. Immunoblotting was performed with respective antibodies to visualize the surface expression of proteins (left panel). Plasma membrane $\mathrm{Ca}^{2+}$ ATPase (PMCA) was used as a loading control. A semiquantitative analysis of the effects of Ala165Pro mutation on the cell surface abundance levels of AVPR2 protein was performed by densitometry (right panel). (D) HEK293 cells were transfected with GFP-tagged WT or Mut AVPR2. At $36 \mathrm{~h}$ after transfection, cells were incubated with $\alpha$-GFP antibody for $1 \mathrm{~h}$ at $4{ }^{\circ} \mathrm{C}$ followed by cell fixation and incubation with Alexa 547 conjugated secondary antibody. The cellular localization of AVPR2 was visualized through immunofluorescence microscopy (40× magnification). Consistent with the immunoblotting results above, the Mut displays less membrane staining when compared to the WT. Green, GFP staining indicates the intracellular and membrane location; red, $\alpha$-GFP staining indicates only the membrane location; yellow, co-localization between the green GFP and red $\alpha$-GFP staining. Values in all bar charts are given in means \pm SEM $(n=3)$. Asterisks indicate significant differences (unpaired $t$-tests with Welch's correction, two-tail): ${ }^{*} p \leq 0.05 ;$ ns, not significant. 
A dDAVP-dependent cAMP production

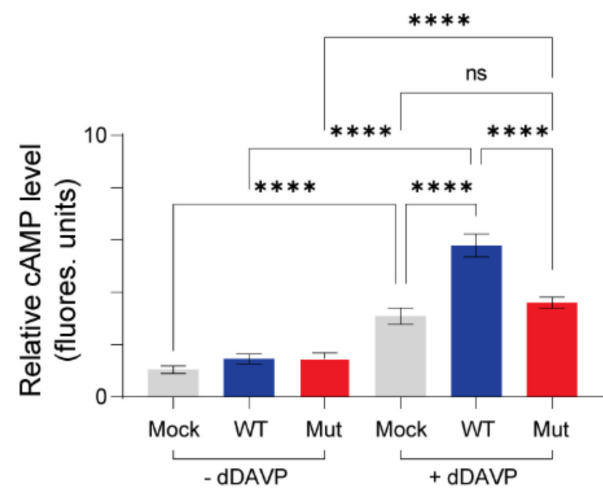

B

dDAVP-dependent PKA Kinase activity

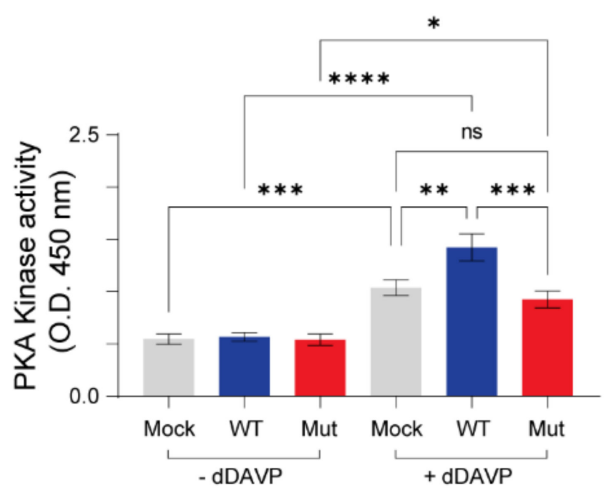

C dDAVP-dependent PKA phosporylation
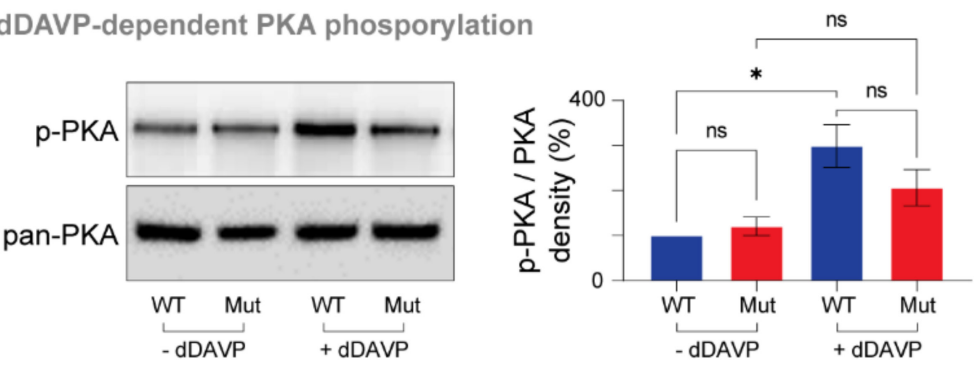

dDAVP-dependent p38MAPK phosporylation
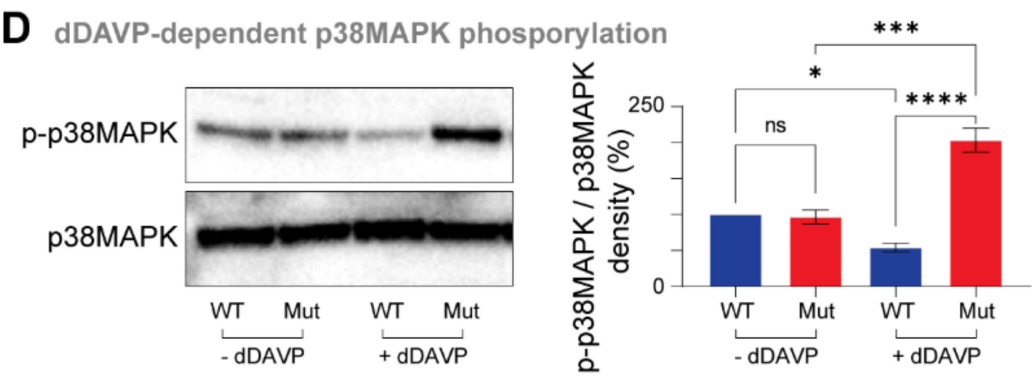

E dDAVP-dependent AQP2 membrane translocation

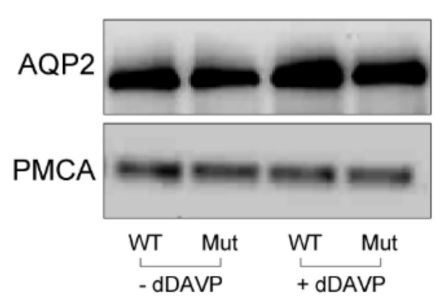

ns
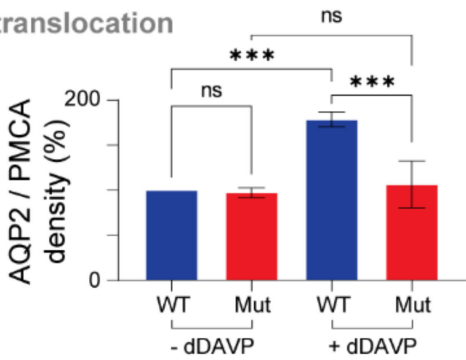

Figure 4. Ala165Pro-AVPR2 mutant impairs the activation of AVP-dependent downstream signaling pathways. (A,B) HEK293 cells were transiently transfected with WT-AVPR2 (WT) or Ala165Pro-AVPR2 (Mut). After $36 \mathrm{~h}$, the cells were stimulated with dimethyl sulfoxide (DMSO) control (-dDAVP) or $1 \mu \mathrm{M}$ dDAVP (+dDAVP) for $10 \mathrm{~min}$ and processed for quantification of intracellular cAMP (A) and PKA kinase activity. (B) The Mut AVPR2 displays lower cAMP and PKA activation levels than the WT. (C,D) WT and Mut AVPR2 expressing cells were stimulated with DMSO control (-dDAVP) or $250 \mathrm{nM}$ dDAVP (+dDAVP) for $60 \mathrm{~min}$. Cells were then harvested, lysed and proteins were analyzed by immunoblotting using antibodies against total and phosphorylated (p-) PKA (C) and p38-MAPK (D) (left panels). Phosphorylated protein abundance was normalized against the respective total proteins and a semiquantitative analysis of the effects of Ala165Pro phosphorylation was done by densitometry (right panels). (E) WT and Mut AVPR2 expressing cells were stimulated with DMSO control (-dDAVP) or $250 \mathrm{nM}$ dDAVP (+dDAVP) for $60 \mathrm{~min}$. Following the treatment, cells were biotinylated using Sulfo-NHS-SS-Biotin and then precipitated with streptavidin-Sepharose beads. Immunoblotting was performed with antibodies against AQP2 to visualize its surface expression (left panel). PMCA was used as a loading control. A semiquantitative analysis of the effects of Ala165Pro mutation on the plasma membrane levels of AVPR2 protein was done by densitometry (right panel). Values in all bar charts are given in means $\pm \operatorname{SEM}(n=3)$. Asterisks indicate significant differences (one-way ANOVA; Tukey multiple comparisons correction): ${ }^{*} p \leq 0.05 ;{ }^{* *} p \leq 0.01$; ${ }^{* * *} p \leq 0.001 ;{ }^{* * *} p \leq 0.001 ;$ ns, not significant.

\section{Discussion}

Here, we identify and functionally characterize a novel AVPR2 missense mutation found in a woman with a history of polyuria and polydipsia. We show that the Ala165Pro substitution in helix 4 of AVPR2 impairs the trafficking, stability and function of AVPR2 and the subsequent translocation of AQP2 to the plasma membrane.

Women who are carriers of $A V P R 2$ mutations are not usually affected by NDI, given the $\mathrm{X}$-linked recessive mode of inheritance. However, in rare cases, this disorder may manifest 
itself due to a mechanism of skewed X-inactivation [38]. X-chromosome inactivation is a natural process that occurs in every female during early embryonic development, by which each cell randomly inactivates either the paternal or the maternal X-chromosome. As a result, females are usually made up of mosaics of cells that statistically will have half of the active X-chromosomes being paternal and the other half maternal. However, this inactivation will sometimes deviate significantly from this 50:50 distribution. If the predominantly expressed $\mathrm{X}$-chromosome happens to have a recessive mutation, then the woman may exhibit a variable phenotype depending on the percentage of cells expressing the mutant gene in the target organ [38]. X-inactivation patterns can differ between tissues and this can explain why this female patient had NDI yet had partial hemodynamic and coagulation responses to AVPR2 stimulation. These findings suggest that the patient had skewed X-inactivation in the kidneys, whereas a more typical random distribution occurred in the liver and vascular endothelium, where factor VIIIc and von Willebrand factor are produced [39]. Unfortunately, we could not experimentally confirm this in our patient due to the inability to obtain tissue samples from these locations.

In terms of mechanism, our cellular assays revealed that the mutant receptor is translated at levels similar to the wild-type, but it fails to reach the cell surface. These results are consistent with the impaired intracellular transport and cell surface expression of AVPR2 caused by other missense mutations (Trp164Ser, Ala165Asp, Ser167Leu, Ser167Thr, Leu170Pro and Gln174Arg) located in the same region as Ala165Pro [36,40-42]. These and other in vitro studies suggest that mutant receptors with distorted transmembrane domains are misfolded, resulting in their retention in the endoplasmic reticulum and proteasomal degradation [12]. On the other hand, defects in the plasma membrane trafficking of AVPR2 mutants result in lower intracellular cAMP levels in recombinant cells [43,44]. However, how changes in the downstream signaling pathways of AVPR2 activation contribute to NDI development is not well established. Thus, understanding the functional consequences of specific mutations may offer new therapeutic avenues for precision medicine approaches. For example, small molecular chaperones have been shown to rescue cell-surface expression and function of some misfolded AVPR2 mutants [45] and cGMP phosphodiesterase inhibitors induce AQP2 membrane insertion independently of the AVPR2 signaling pathways [46].

Agonist mediated stimulation of AVPR2 induces the cAMP signaling cascade in renal collecting duct principal cells that trigger the translocation of AQP2 to the apical membrane, resulting in water reabsorption. In our experiments, the mutant Ala165Pro-AVPR2 showed reduced intracellular cAMP levels and cAMP-mediated PKA activation upon stimulation with dDAVP, consistent with previous studies [47-49]. Our experiments further revealed that in the WT-AVPR2, but not in the Ala165Pro-AVPR2, this cAMP-mediated PKA activation leads to a PKA phosphorylation at Thr197 and subsequent inhibition of p38-MAPK and decreased Ser261 phosphorylation, in line with previous results in fibroblasts and HeLa cells [50]. In contrast, in the Ala165Pro-AVPR2, cAMP-mediated PKA activation upon dDAVP stimulation leads to an increase in p38-MAPK phosphorylation, suggesting that production of intracellular cAMP controls the downstream activation of PKA and PKA dependent p38-MAPK inhibition.

Vasopressin regulates AQP2 protein abundance by increasing the intracellular level of cAMP, which activates PKA-mediated inhibition of p38-MAPK and p38-MAPK-dependent regulation of the proteasomal degradation of AQP2 [51,52]. Moreover, vasopressin increases the abundance of AQP2 in the plasma membrane by regulating the trafficking of AQP2 between the intracellular vesicles and the apical plasma membrane. Our experiments show that the Ala165Pro-AVPR2 substitution leads to a reduction in the membrane levels of AQP2 in response to dDAVP compared to the WT-AVPR2. These data suggest that the impaired vasopressin-dependent cAMP signaling leads to defects in AQP2 transcription, translation, stability and trafficking to the membrane, which ultimately manifests in the patient as an inability to concentrate urine.

In summary, we identified a rare case of a woman affected with NDI and showed that her condition was caused by a novel missense mutation of the AVPR2 gene, leading 
to impaired trafficking, stability and function of AVPR2. Our study extends the known mutational spectrum of the AVPR2 gene, the key role of $\mathrm{X}$-inactivation mosaicism in NDI in female patients and further contributes to the understanding of the pathogenesis of this disorder.

Author Contributions: S.S. performed experiments, analyzed data and wrote the initial version of the manuscript. D.R. performed experiments and analyzed data. N.K. performed experiments, analyzed data and helped write the initial version of the manuscript. K.A.F. analyzed data and commented on the final version of the manuscript. L.R.S. and M.C.L. conceived and supervised the project, analyzed data and wrote the final version of the manuscript. All authors have read and agreed to the published version of the manuscript.

Funding: This work was supported by the Portuguese Foundation for Science and Technology (FCT, project grant UIDB/00709/2020), by Programa Operacional Regional do Centro (project grant CENTRO-08-5864-FSE-000039) and by Sidra Medicine (grant number SDR400038) - a member of the Qatar Foundation.

Institutional Review Board Statement: The study was conducted in accordance with the Declaration of Helsinki, and approved by the Ethics Committee of the Faculty of Health Sciences, University of Beira Interior, Portugal (reference CE-FCS-2013-017, approved 12 June 2013).

Informed Consent Statement: Informed consent was obtained from all subjects involved in the study.

Data Availability Statement: The data presented in this study are available in Figures 1-4. The raw data presented in this study are available on request from the corresponding author. Requests for materials should be addressed to L.R.S.

Acknowledgments: We would like to thank the Saraiva Lab members for their valuable feedback on this manuscript.

Conflicts of Interest: All authors declare that they have no competing interests. The funders had no role in study design, data collection, data analysis, decision to publish, or preparation of the manuscript.

\section{References}

1. Rondon-Berrios, H.; Berl, T. Physiology and Pathophysiology of Water Homeostasis. Front. Horm. Res. 2019, 52, 8-23. [PubMed]

2. Christ-Crain, M.; Bichet, D.G.; Fenske, W.K.; Goldman, M.B.; Rittig, S.; Verbalis, J.G.; Verkman, A.S. Diabetes insipidus. Nat. Rev. Dis. Prim. 2019, 5, 54. [CrossRef] [PubMed]

3. Kavanagh, C.; Uy, N.S. Nephrogenic Diabetes Insipidus. Pediatr. Clin. N. Am. 2019, 66, 227-234. [CrossRef]

4. Pan, Y.; Metzenberg, A.; Das, S.; Jing, B.; Gitschier, J. Mutations in the V2 vasopressin receptor gene are associated with X-linked nephrogenic diabetes insipidus. Nat. Genet. 1992, 2, 103-106. [CrossRef] [PubMed]

5. Rosenthal, W.; Seibold, A.; Antaramian, A.; Lonergan, M.; Arthus, M.F.; Hendy, G.N.; Birnbaumer, M.; Bichet, D.-G. Molecular identification of the gene responsible for congenital nephrogenic diabetes insipidus. Nature 1992, 359, 233-235. [CrossRef]

6. Van den Ouweland, A.M.; Dreesen, J.C.; Verdijk, M.; Knoers, N.V.; Monnens, L.A.; Rocchi, M.; van Oost, B.A. Mutations in the vasopressin type 2 receptor gene (AVPR2) associated with nephrogenic diabetes insipidus. Nat. Genet. 1992, 2, 99-102. [CrossRef]

7. Bichet, D.G. Genetics in Endocrinology Pathophysiology, diagnosis and treatment of familial nephrogenic diabetes insipidus. Eur. J. Endocrinol. 2020, 183, R29-R40. [CrossRef]

8. D'Alessandri-Silva, C.; Carpenter, M.; Ayoob, R.; Barcia, J.; Chishti, A.; Constantinescu, A.; Dell, K.M.; Goodwin, J.; Hashmat, S.; Iragorri, S.; et al. Diagnosis, Treatment, and Outcomes in Children With Congenital Nephrogenic Diabetes Insipidus: A Pediatric Nephrology Research Consortium Study. Front. Pediatr. 2019, 7, 550. [CrossRef]

9. Sharma, S.; Ashton, E.; Iancu, D.; Arthus, M.F.; Hayes, W.; Van't Hoff, W.; Kleta, R.; Bichet, D.G.; Bockenhauer, D. Long-term outcome in inherited nephrogenic diabetes insipidus. Clin. Kidney J. 2019, 12, 180-187. [CrossRef]

10. Birnbaumer, M.; Seibold, A.; Gilbert, S.; Ishido, M.; Barberis, C.; Antaramian, A.; Brabet, P.; Rosenthal, W. Molecular cloning of the receptor for human antidiuretic hormone. Nature 1992, 357, 333-335. [CrossRef]

11. Seibold, A.; Brabet, P.; Rosenthal, W.; Birnbaumer, M. Structure and chromosomal localization of the human antidiuretic hormone receptor gene. Am. J. Hum. Genet. 1992, 51, 1078-1083. [PubMed]

12. Spanakis, E.; Milord, E.; Gragnoli, C. AVPR2 variants and mutations in nephrogenic diabetes insipidus: Review and missense mutation significance. J. Cell. Physiol. 2008, 217, 605-617. [CrossRef] [PubMed]

13. Ding, C.; Beetz, R.; Rittner, G.; Bartsch, O. A female with X-linked Nephrogenic diabetes insipidus in a family with inherited central diabetes Insipidus: Case report and review of the literature. Am. J. Med. Genet. Part A 2020, 182, 1032-1040. [CrossRef] [PubMed] 
14. Bichet, D.G.; Razi, M.; Lonergan, M.; Arthus, M.F.; Papukna, V.; Kortas, C.; Barjon, J.-N. Hemodynamic and coagulation responses to 1-desamino[8-D-arginine] vasopressin in patients with congenital nephrogenic diabetes insipidus. N. Engl. J. Med. 1988, 318, 881-887. [CrossRef]

15. Fakhro, K.A.; Robay, A.; Rodrigues-Flores, J.L.; Mezey, J.G.; Al-Shakaki, A.A.; Chidiac, O.; Stadler, D.; Malek, J.A.; Imam, A.B.; Sheikh, A.; et al. Point of Care Exome Sequencing Reveals Allelic and Phenotypic Heterogeneity Underlying Mendelian disease in Qatar. Hum. Mol. Genet. 2019, 28, 3970-3981. [CrossRef]

16. Karczewski, K.J.; Francioli, L.C.; Tiao, G.; Cummings, B.B.; Alfoldi, J.; Wang, Q.; Collins, R.L.; Laricchia, K.M.; Ganna, A.; Birnbaum, D.P.; et al. The mutational constraint spectrum quantified from variation in 141,456 humans. Nature 2020, 581, 434-443. [CrossRef]

17. Ritchie, G.R.; Flicek, P. Computational approaches to interpreting genomic sequence variation. Genome Med. 2014, 6, 87. [CrossRef]

18. Kopanos, C.; Tsiolkas, V.; Kouris, A.; Chapple, C.E.; Albarca Aguilera, M.; Meyer, R.; Massouras, A. VarSome: The human genomic variant search engine. Bioinformatics 2019, 35, 1978-1980. [CrossRef]

19. Richards, S.; Aziz, N.; Bale, S.; Bick, D.; Das, S.; Gastier-Foster, J.; Grody, W.W.; Hegde, M.; Lyon, E.; Spector, E.; et al. Standards and guidelines for the interpretation of sequence variants: A joint consensus recommendation of the American College of Medical Genetics and Genomics and the Association for Molecular Pathology. Genet. Med. 2015, 17, 405-424. [CrossRef] [PubMed]

20. Macion-Dazard, R.; Callahan, N.; Xu, Z.; Wu, N.; Thibonnier, M.; Shoham, M. Mapping the binding site of six nonpeptide antagonists to the human V2-renal vasopressin receptor. J. Pharmacol. Exp. Ther. 2006, 316, 564-571. [CrossRef] [PubMed]

21. Thibonnier, M.; Graves, M.K.; Wagner, M.S.; Chatelain, N.; Soubrier, F.; Corvol, P.; Willard, H.F.; Jeunemaitre, X. Study of $\mathrm{V}(1)$-vascular vasopressin receptor gene microsatellite polymorphisms in human essential hypertension. J. Mol. Cell. Cardiol. 2000, 32, 557-564. [CrossRef] [PubMed]

22. Decaux, G.; Soupart, A.; Vassart, G. Non-peptide arginine-vasopressin antagonists: The vaptans. Lancet 2008, $371,1624-1632$. [CrossRef]

23. Suno, R.; Kimura, K.T.; Nakane, T.; Yamashita, K.; Wang, J.; Fujiwara, T.; Yamanaka, Y.; Im, D.; Horita, S.; Tsujimoto, H.; et al Crystal Structures of Human Orexin 2 Receptor Bound to the Subtype-Selective Antagonist EMPA. Structure 2018, 26, 7-19.e5. [CrossRef] [PubMed]

24. Rappas, M.; Ali, A.A.E.; Bennett, K.A.; Brown, J.D.; Bucknell, S.J.; Congreve, M.; Cooke, R.M.; Cseke, G.; De Graaf, C.; Doré, A.S.; et al. Comparison of Orexin 1 and Orexin 2 Ligand Binding Modes Using X-ray Crystallography and Computational Analysis. J. Med. Chem. 2020, 63, 1528-1543. [CrossRef]

25. Waltenspuhl, Y.; Schoppe, J.; Ehrenmann, J.; Kummer, L.; Pluckthun, A. Crystal structure of the human oxytocin receptor. Sci. Adv. 2020, 6, eabb5419. [CrossRef]

26. Staus, D.P.; Hu, H.; Robertson, M.J.; Kleinhenz, A.L.W.; Wingler, L.M.; Capel, W.D.; Latorraca, N.R.; Lefkowitz, R.J.; Skiniotis, G. Structure of the M2 muscarinic receptor-beta-arrestin complex in a lipid nanodisc. Nature 2020, 579, 297-302. [CrossRef]

27. Hellmann, J.; Drabek, M.; Yin, J.; Gunera, J.; Proll, T.; Kraus, F.; Langmead, C.J.; Hübner, H.; Weikert, D.; Kolb, P.; et al. Structurebased development of a subtype-selective orexin 1 receptor antagonist. Proc. Natl. Acad. Sci. USA 2020, 117, 18059-18067. [CrossRef]

28. Waterhouse, A.; Bertoni, M.; Bienert, S.; Studer, G.; Tauriello, G.; Gumienny, R.; Heer, F.T.; De Beer, T.A.P.; Rempfer, C.; Bordoli, L.; et al SWISS-MODEL: Homology modelling of protein structures and complexes. Nucleic Acids Res. 2018, 46, W296-W303. [CrossRef]

29. Studer, G.; Rempfer, C.; Waterhouse, A.M.; Gumienny, R.; Haas, J.; Schwede, T. QMEANDisCo-distance constraints applied on model quality estimation. Bioinformatics 2020, 36, 2647. [CrossRef]

30. Zaki, O.K.; Krishnamoorthy, N.; El Abd, H.S.; Harche, S.A.; Mattar, R.A.; Al Disi, R.S.; Nofal, M.Y.; El Bekay, R.; Ahmed, A.K.; Doss, C.G.P.; et al. Two patients with Canavan disease and structural modeling of a novel mutation. Metab. Brain Dis. 2017, 32, 171-177. [CrossRef]

31. Gajendrarao, P.; Krishnamoorthy, N.; Kassem, H.; Moharem-Elgamal, S.; Cecchi, F.; Olivotto, I.; Yacoub, M.H. Molecular modeling of disease causing mutations in domain C1 of cMyBP-C. PLoS ONE 2013, 8, e59206. [CrossRef] [PubMed]

32. Selvaraj, S.; Sun, Y.; Sukumaran, P.; Singh, B.B. Resveratrol activates autophagic cell death in prostate cancer cells via downregulation of STIM1 and the mTOR pathway. Mol. Carcinog. 2016, 55, 818-831. [CrossRef] [PubMed]

33. Tahtaoui, C.; Balestre, M.N.; Klotz, P.; Rognan, D.; Barberis, C.; Mouillac, B.; Hibert, M. Identification of the binding sites of the SR49059 nonpeptide antagonist into the V1a vasopressin receptor using sulfydryl-reactive ligands and cysteine mutants as chemical sensors. J. Biol. Chem. 2003, 278, 40010-40019. [CrossRef]

34. Cordes, F.S.; Bright, J.N.; Sansom, M.S. Proline-induced distortions of transmembrane helices. J. Mol. Biol. 2002, 323, 951-960. [CrossRef]

35. Grimm, C.; Cuajungco, M.P.; van Aken, A.F.; Schnee, M.; Jors, S.; Kros, C.J.; Ricci, A.J.; Heller, S. A helix-breaking mutation in TRPML3 leads to constitutive activity underlying deafness in the varitint-waddler mouse. Proc. Natl. Acad. Sci. USA 2007, 104 19583-19588. [CrossRef]

36. Schulz, A.; Sangkuhl, K.; Lennert, T.; Wigger, M.; Price, D.A.; Nuuja, A.; Grüters, A.; Schultz, G.; Schöneberg, T. Aminoglycoside pretreatment partially restores the function of truncated $\mathrm{V}(2)$ vasopressin receptors found in patients with nephrogenic diabetes insipidus. J. Clin. Endocrinol. Metab. 2002, 87, 5247-5257. [CrossRef]

37. Boone, M.; Deen, P.M. Physiology and pathophysiology of the vasopressin-regulated renal water reabsorption. Pflug. Arch. 2008, 456, 1005-1024. [CrossRef] 
38. Migeon, B.R. X inactivation, female mosaicism, and sex differences in renal diseases. J. Am. Soc. Nephrol. 2008, 19, 2052-2059. [CrossRef] [PubMed]

39. Moses, A.M.; Sangani, G.; Miller, J.L. Proposed cause of marked vasopressin resistance in a female with an X-linked recessive V2 receptor abnormality. J. Clin. Endocrinol. Metab. 1995, 80, 1184-1186. [PubMed]

40. Oksche, A.; Schulein, R.; Rutz, C.; Liebenhoff, U.; Dickson, J.; Muller, H.; Birnbaumer, M.; Rosenthal, W. Vasopressin V2 receptor mutants that cause X-linked nephrogenic diabetes insipidus: Analysis of expression, processing, and function. Mol. Pharmacol. 1996, 50, 820-828.

41. Nejsum, L.N.; Christensen, T.M.; Robben, J.H.; Milligan, G.; Deen, P.M.; Bichet, D.G.; Levin, K. Novel mutation in the AVPR2 gene in a Danish male with nephrogenic diabetes insipidus caused by ER retention and subsequent lysosomal degradation of the mutant receptor. NDT Plus 2011, 4, 158-163. [CrossRef]

42. Boselt, I.; Tramma, D.; Kalamitsou, S.; Niemeyer, T.; Nykanen, P.; Graf, K.J.; Krude, H.; Marenzi, K.S.; Di Candia, S.; Schöneberg, T.; et al. Functional characterization of novel loss-of-function mutations in the vasopressin type 2 receptor gene causing nephrogenic diabetes insipidus. Nephrol. Dial. Transplant. 2012, 27, 1521-1528. [CrossRef]

43. Carpentier, E.; Greenbaum, L.A.; Rochdi, D.; Abrol, R.; Goddard, W.A.; Bichet, D.G., III; Bouvier, M. Identification and characterization of an activating F229V substitution in the V2 vasopressin receptor in an infant with NSIAD. J. Am. Soc. Nephrol. 2012, 23, 1635-1640. [CrossRef]

44. Tiulpakov, A.; White, C.W.; Abhayawardana, R.S.; See, H.B.; Chan, A.S.; Seeber, R.M.; Heng, J.I.; Dedov, I.; Pavlos, N.J.; Pfleger, K.D. Mutations of Vasopressin Receptor 2 Including Novel L312S Have Differential Effects on Trafficking. Mol. Endocrinol. 2016, 30, 889-904. [CrossRef] [PubMed]

45. Morello, J.P.; Salahpour, A.; Laperriere, A.; Bernier, V.; Arthus, M.F.; Lonergan, M.; Petäjä-Repo, U.; Angers, S.; Morin, D.; Bichet, D.G.; et al. Pharmacological chaperones rescue cell-surface expression and function of misfolded V2 vasopressin receptor mutants. J. Clin. Investig. 2000, 105, 887-895. [CrossRef] [PubMed]

46. Bouley, R.; Pastor-Soler, N.; Cohen, O.; McLaughlin, M.; Breton, S.; Brown, D. Stimulation of AQP2 membrane insertion in renal epithelial cells in vitro and in vivo by the cGMP phosphodiesterase inhibitor sildenafil citrate (Viagra). Am. J. Physiol. Renal Physiol. 2005, 288, F1103-F11012. [CrossRef] [PubMed]

47. Miller, R.L.; Sandoval, P.C.; Pisitkun, T.; Knepper, M.A.; Hoffert, J.D. Vasopressin inhibits apoptosis in renal collecting duct cells. Am. J. Physiol. Renal Physiol. 2013, 304, F177-F188. [CrossRef]

48. Goel, M.; Zuo, C.D.; Schilling, W.P. Role of cAMP/PKA signaling cascade in vasopressin-induced trafficking of TRPC3 channels in principal cells of the collecting duct. Am. J. Physiol. Renal Physiol. 2010, 298, F988-F996. [CrossRef]

49. Prosperi, F.; Suzumoto, Y.; Marzuillo, P.; Costanzo, V.; Jelen, S.; Iervolino, A.; Guarino, S.; La Manna, A.; Del Giudice, E.M.; Perna, A.F.; et al. Characterization of five novel vasopressin V2 receptor mutants causing nephrogenic diabetes insipidus reveals a role of tolvaptan for M272R-V2R mutation. Sci. Rep. 2020, 10, 16383. [CrossRef]

50. Zhang, J.; Bui, T.N.; Xiang, J.; Lin, A. Cyclic AMP inhibits p38 activation via CREB-induced dynein light chain. Mol. Cell. Biol. 2006, 26, 1223-1234. [CrossRef]

51. Nedvetsky, P.I.; Tabor, V.; Tamma, G.; Beulshausen, S.; Skroblin, P.; Kirschner, A.; Mutig, K.; Boltzen, M.; Petrucci, O.; Vossenkämper, A.; et al. Reciprocal regulation of aquaporin-2 abundance and degradation by protein kinase A and p38-MAP kinase. J. Am. Soc. Nephrol. 2010, 21, 1645-1656. [CrossRef] [PubMed]

52. Christensen, B.M.; Marples, D.; Jensen, U.B.; Frokiaer, J.; Sheikh-Hamad, D.; Knepper, M.; Nielsen, S. Acute effects of vasopressin V2-receptor antagonist on kidney AQP2 expression and subcellular distribution. Am. J. Physiol. 1998, 275, F285-F297. [CrossRef] [PubMed] 